\title{
La innovación como cultura organizacional sustentada en procesos humanos
}

\author{
Flores Urbáez, Matilde*
}

\section{Resumen}

La innovación ha sido analizada por gobiernos y organismos internacionales en décadas recientes. Producto de esta reflexión y del estudio de experiencias, se concluye que no solo debe asociarse a la innovación proveniente de la actividad científica y tecnológica de punta y que tampoco existe correlación absoluta entre la capacidad de innovación y el presupuesto asignado para ello. Lo que sí es determinante para estimular este proceso son organizaciones flexibles y gerentes con actitud abierta a explorar nuevas experiencias que potencien el talento innovador de la gente en todos los niveles de la organización. Por lo anterior, presentamos un análisis teórico- reflexivo de la innovación como cultura de trabajo sustentada principalmente en procesos humanos. Se concluye que para implementar exitosamente la cultura de innovación en todos los niveles de la organización es importante pensar en red, tener conciencia de que se forma parte de otros sistemas con los que debemos cooperar para innovar, y de la importancia de valores que permitan incorporar la cultura de innovación en la organización de manera armónica y sustentable.

Palabras clave: Innovación; cultura organizacional; procesos humanos; innovación no tecnológica

\section{Innovation as a human processes sustained organizational culture.}

\section{Abstract}

Innovation has been analyzed by governments and international organisms in recent decades. Product of this reflection and experiences studies, it is concluded that it should not only be associated to innovation from the scientific and sharp edge technologic activity and that there is

Recibido: 7.02 .15 . Aceptado: 20.05 .15

* Profesora Titular. Departamento de Ciencias Humanas. Facultad Experimental de Ciencias. Universidad del Zulia. Doctora en Ciencias Sociales, mención Gerencia. E-mail: floresurbaez8@ gmail.com 
no absolute correlation between the innovation capacity and the budget assigned for it. What are crucial to stimulate this process are the flexible organizations and managers with open attitude to explore new experiences that potentiate the peoples innovative talent across all the levels of the organization. By the foregoing, a theoretic reflective analysis of the innovation as a working culture mainly sustained in human processes is presented. It is concluded that in order to implement successfully the innovation culture through every level of the organization is important to think on the network, to be aware that it is part of other systems among which we must cooperate to innovate, and of the values importance that allows to incorporate to the organization the innovation culture in a harmonic and sustainable manner.

Key Words: Innovation; organizational culture; human processes; non technological innovation.

\section{Introducción}

El desarrollo de la humanidad está asociado con la actitud innovadora de sus protagonistas, individuales o colectivos. Esta cualidad presente en los pensadores de la antigua Grecia es un ejemplo de ello. A pesar del status quo de su época, se atrevieron a intentar explicar de manera racional y no a través de lo sobrenatural, el comportamiento del ser humano y de la naturaleza, hechos que marcaron la configuración del pensamiento y la ciencia occidental, junto con la influencia de otras culturas antiguas como la árabe y la china.

En la realidad actual, impactada por crisis de diversa índole (energética, financiera, social, política, de valores, climática, ambiental, guerras, entre otras), es incuestionable la necesidad de innovar, tanto para crear oportunidades de progreso como para concebir nuevos caminos para alcanzarlo. El proceso de innovación en una organización puede influir, directa o indirectamente, en su sector de actividad y en el país donde se encuentre porque implica, en términos de Schumpeter, un proceso de "destrucción creativa", es decir, destrucción de lo antiguo para crear continua y sistemáticamente elementos nuevos que permitan a la organización crear condiciones que refuercen su capacidad de adaptación al medio dinámico y favorezcan su capacidad de innovar.

Los individuos y las organizaciones, desde las más simples a las más complejas, han innovado, de manera consciente o no, para mantenerse en el tiempo. Por tal motivo, y aunado a la diversidad de los procesos organizacionales, el significado del término innovación también ha tenido que ampliarse aunque de manera progresiva y concebirse desde distintas perspectivas en función de lo que se innova.

Este concepto ha sido foco de análisis en eventos especializados y en mesas de discusión por parte de gobiernos y organismos internacionales, donde producto de la reflexión y estudio de experiencias en distintas partes del mundo, se ha llegado a la conclusión de que la innovación no solo debe asociarse a la innovación tecnológica proveniente de la actividad científica de punta y que tampoco existe correlación absoluta entre la capacidad para la innovación de las organizaciones y el presupuesto asignado para ello, porque innovar no necesariamente requiere de grandes presupuestos (González, 2013).

Lo que sí consideramos determinante para estimular este interesante proceso, entre otros aspectos, son organizaciones flexibles dirigidas por gerentes con cualidades 
como la actitud abierta a explorar nuevas experiencias que potencien el talento innovador de su gente; visión de futuro; disponibilidad de tiempo para reflexionar junto con el personal sobre posibles innovaciones; visualización de los errores como oportunidades de aprendizaje y capacidad para asumir riesgos calculados. Todo lo anterior implica que se requiere de una cultura organizacional donde la innovación forma parte de la cotidianidad; lo que favorecerá, pudiéramos decir de forma casi absoluta, que la organización, independientemente de su actividad, naturaleza y tamaño, se posicione en su entorno como una referencia de éxito teniendo como principal activo el conocimiento y la capacidad de innovación de su gente.

Por todo lo anterior y considerando que la actitud innovadora de la gente en toda la estructura de la organización es determinante para el éxito de la misma, presentamos en este trabajo un análisis teórico y reflexivo de la innovación, no desde el punto de vista técnicoeconómico, sino como cultura de trabajo sustentada principalmente en procesos humanos.

\section{Evolución teórico conceptual de la innovación}

En este trabajo tomaremos como referencia para describir brevemente la evolución del enfoque de la innovación en los países desarrollados, la plasmada en el Manual de Oslo, documento publicado por la Organización para la Cooperación y el Desarrollo Económico (OCDE, 2006) y la Oficina de Estadística de las Comunidades Europeas (EUROSTAT). El mismo es considerado un referente conceptual sobre asuntos de innovación. Su uso se ha generalizado a nivel mundial incluso en Latinoamérica debido a la existencia de un interés generalizado en la región por el estudio de la innovación.

Tomaremos como referencia para describir la evolución del enfoque de la innovación en Latinoamérica las memorias de la Asociación Latinoiberoamericana de Gestión Tecnológica (ALTEC), institución privada internacional sin fines de lucro, creada en 1984 con el propósito de vincular organizaciones (públicas y privadas), profesionales (académicos y no académicos) y otros interesados en el estudio y ejercicio de la gestión de la tecnología con el fin de realizar actividades de cooperación e intercambio de conocimientos en esta área. Se considera que la ALTEC ha contribuido a generar un pensamiento latino-iberoamericano sobre problemas relacionados con la gestión de la tecnología y la innovación:

\section{a) En los países europeos}

En la primera y segunda edición del Manual de Oslo (1992 y 1997 respectivamente) se utilizó la definición de innovación desde la perspectiva de la tecnología de proceso y de producto. Esto supuso una focalización en el desarrollo tecnológico de nuevos productos y nuevas técnicas de producción para las empresas, sobre todo las de manufactura. La discusión sobre innovación organizativa y sobre la innovación no tecnológica fue incluida en un anexo (OCDE, 2006). La tercera y última edición del Manual (2006) se amplió el marco de la innovación de tres maneras las cuales fueron incorporadas en esa edición:

- Importancia de los vínculos con otras empresas e instituciones en el proceso de innovación: El énfasis en los vínculos interorganizacionales se debe a que éstos favorecen el flujo 
de conocimientos para el desarrollo y difusión de las innovaciones como por ejemplo, a través de la participación en proyectos conjuntos de innovación.

- Importancia de la innovación en sectores de baja intensidad en I+D como los servicios y las industrias con escaso contenido tecnológico: La innovación en las empresas de servicios es "mucho menos formal, más incremental y menos tecnológica".

- Inclusión de dos tipos adicionales de innovación: La organizativa y la de mercadotécnia que junto con el uso de una definición más amplia de innovación, favoreció la inclusión de más empresas como innovadoras. Se considera que las innovaciones organizativas, pueden mejorar la calidad y la eficiencia del trabajo, favorecer el intercambio de información, dotar a las empresas de una mayor capacidad de aprendizaje y de utilización de nuevos conocimientos y tecnologías y ayudar a la organización a beneficiarse de otros tipos de innovación.

\section{b. En los países \\ latinoiberoamericanos}

En el cuadro 1 presentamos las ediciones del seminario de Altec iniciando desde 1991 por ser el año más cercano a la primera edición del Manual de Oslo, con los respectivos temas centrales y ejes temáticos con el fin de realizar un breve análisis de la evolución del interés e Iberoamérica por el abordaje de la relación innovación-procesos humanos.

\section{Cuadro 1}

\section{Evolución del abordaje explícito de la relación innovación- procesos humanos en los seminarios de Altec en sus ejes temáticos}

\begin{tabular}{|c|c|c|c|}
\hline Año & Lugar & Temas centrales & $\begin{array}{l}\text { Ejes o sub ejes temáticos } \\
\text { explícitamente relacionados con } \\
\text { la relación innovación-procesos } \\
\text { humanos }\end{array}$ \\
\hline 1991 & $\begin{array}{l}\text { Caracas- } \\
\text { Venezuela }\end{array}$ & $\begin{array}{l}\text { Competitividad del sector } \\
\text { productivo, reconversión industrial } \\
\text { y tecnología }\end{array}$ & Ninguno \\
\hline 1993 & $\begin{array}{l}\text { Bogotá- } \\
\text { Colombia }\end{array}$ & $\begin{array}{l}\text { Competitividad y Globalización; } \\
\text { Nuevas Tecnologías; Gerencia de } \\
\text { la Innovación Tecnológica }\end{array}$ & $\begin{array}{c}\text { Gerencia de la innovación tecnológica } \\
\text { y competitividad }\end{array}$ \\
\hline 1995 & $\begin{array}{l}\text { Concepción- } \\
\text { Chile }\end{array}$ & $\begin{array}{l}\text { Gestión tecnológica, competitividad } \\
\text { y empleo }\end{array}$ & $\begin{array}{l}\text { Experiencias sobre educación en el } \\
\text { desarrollo de la capacidad innovadora }\end{array}$ \\
\hline 1997 & $\begin{array}{l}\text { La Habana- } \\
\text { Cuba }\end{array}$ & $\begin{array}{l}\text { Innovación, competitividad y } \\
\text { desarrollo sustentable }\end{array}$ & $\begin{array}{l}\text { Ejes temáticos no definidos en las } \\
\text { memorias }\end{array}$ \\
\hline 1999 & $\begin{array}{l}\text { Valencia- } \\
\text { España }\end{array}$ & $\begin{array}{c}\text { Innovación Tecnológica para el } \\
\text { Tercer Milenio }\end{array}$ & $\begin{array}{l}\text { Los impactos de la innovación: } \\
\text { Indicadores y gestión del conocimiento }\end{array}$ \\
\hline
\end{tabular}

Fuente: Elaboración propia con base en las memorias de Altec 


\section{Cont... Cuadro 1}

\begin{tabular}{|c|c|c|c|}
\hline Año & Lugar & Temas centrales & $\begin{array}{l}\text { Ejes o sub ejes temáticos } \\
\text { explícitamente relacionados con } \\
\text { la relación innovación-procesos } \\
\text { humanos }\end{array}$ \\
\hline 2001 & $\begin{array}{c}\text { San José-Costa } \\
\text { Rica }\end{array}$ & $\begin{array}{l}\text { Innovación Tecnológica en la } \\
\text { Economía del Conocimiento }\end{array}$ & $\begin{array}{l}\text { Gestión del conocimiento para la } \\
\text { innovación }\end{array}$ \\
\hline 2003 & $\begin{array}{l}\text { México DF- } \\
\text { México }\end{array}$ & $\begin{array}{c}\text { Conocimiento, Innovación y } \\
\text { Competitividad: Los desafíos de la } \\
\text { globalización }\end{array}$ & $\begin{array}{l}\text { Gestión y transferencia del } \\
\text { conocimiento. } \\
\text { Estrategias de aprendizaje y } \\
\text { construcción de capacidades de } \\
\text { innovación. }\end{array}$ \\
\hline 2005 & Salvador-Brasil & $\begin{array}{l}\text { Innovación Tecnológica, } \\
\text { Cooperación y Desarrollo }\end{array}$ & $\begin{array}{l}\text { La figura del gerente y sus } \\
\text { competencias. } \\
\text { Clima para la innovación. }\end{array}$ \\
\hline 2007 & $\begin{array}{l}\text { Buenos Aires- } \\
\text { Argentina }\end{array}$ & Producción, empleo e inclusión & $\begin{array}{l}\text { El conocimiento como herramienta de } \\
\qquad \text { la innovación. }\end{array}$ \\
\hline 2009 & $\begin{array}{l}\text { Cartagena- } \\
\text { Colombia }\end{array}$ & $\begin{array}{l}\text { Innovación y creatividad para el } \\
\text { desarrollo sostenible }\end{array}$ & $\begin{array}{l}\text { El conocimiento como herramienta de } \\
\qquad \text { la innovación }\end{array}$ \\
\hline 2011 & Lima-Perú & $\begin{array}{c}\text { Innovación para el crecimiento } \\
\text { sostenible }\end{array}$ & $\begin{array}{l}\text { La innovación y la gestión del } \\
\text { conocimiento } \\
\text { La vinculación entre los actores para la } \\
\text { innovación }\end{array}$ \\
\hline 2013 & Porto-Portugal & $\begin{array}{l}\text { Políticas y gestión de ciencia } \\
\text { y tecnología en el contexto } \\
\text { latinoiberoamericano }\end{array}$ & $\begin{array}{c}\text { Factores humanos y culturales en el } \\
\text { desarrollo de la innovación científica y } \\
\text { tecnológica }\end{array}$ \\
\hline 2015 & $\begin{array}{l}\text { Porto Alegre- } \\
\text { Brasil }\end{array}$ & $\begin{array}{l}\text { Innovación más allá de la } \\
\text { tecnología" }\end{array}$ & $\begin{array}{l}\text { Ecosistema de innovación } \\
\text { Cooperación y redes para la } \\
\text { innovación }\end{array}$ \\
\hline
\end{tabular}

Fuente: Elaboración propia con base en las memorias de Altec

Se observa que los temas centrales del evento estuvieron hasta el año 2001 mayormente orientados hacia la innovación tecnológica pero expresando en algunos ejes temáticos el foco de lo humano en el proceso innovador. Es a partir de la edición de 2003 realizada en México cuando se incorpora de manera explícita como tema central del seminario la innovación sin el adjetivo "tecnológica", dando apertura al análisis de otro tipo de innovaciones. El foco de atención explícito hacia el conocimiento como eje temático se evidencia en la edición de 2001 en Costa Rica. En 2005 en Brasil se retoma como tema central la innovación tecnológica, pero en 2007 en Argentina el tema central consideramos que en sí mismo fue innovador "Producción, empleo e inclusión", lo que motivó a los participantes a relacionar explícitamente los temas de gestión de la tecnología e innovación con los temas centrales orientados a lo 
social, incorporando como uno de los ejes temáticos "El conocimiento como herramienta de la innovación".

En el evento de 2009 realizado en Cartagena, Colombia, se incluye nuevamente como eje temático "El conocimiento como herramienta de la innovación" y aparece por primera vez como eje temático la "innovación social". En Altec 2011 llevado a cabo en Lima, Perú, se fortalecen los ejes temáticos que expresan el interés de asociar la innovación con procesos humanos, estos ejes son "La innovación y la gestión del conocimiento" y "La vinculación entre los actores para la innovación". Nuevamente se trata como eje temático la innovación social.

En el evento realizado en Porto, Portugal en 2013, aun cuando el tema central fue un tanto genérico (Políticas y gestión de ciencia y tecnología en el contexto latinoiberoamericano), encontramos un eje temático mucho más explícito que resalta la relación entre la innovación y lo humano aun cuando retoman la innovación tecnológica que había sido dejada un poco atrás en ediciones anteriores. La relación de la innovación con lo humano se evidencia en el eje temático "Factores humanos y culturales en el desarrollo de la innovación científica y tecnológica". Finalmente, asumimos que motivados por estos y otros elementos que hemos tratado, la última edición de Altec que se llevará a cabo en octubre de 2015 en Porto Alegre, Brasil, tendrá como tema central "Innovación más allá de la tecnología" y revisando los ejes temáticos encontramos dos muy interesantes: "Ecosistema de innovación" y "Cooperación y redes para la innovación", los cuales nos demuestran el interés de la Altec por profundizar en el estudio el rol medular del ser humano y de sus interacciones para fortalecer la cultura de innovación en la región.

Al hablar de ecosistemas de innovación podemos asociarlo a dos aspectos: el primero, incorporar innovaciones en los procesos organizacionales asociados tanto a la creación de valores como la incorporación de aspectos tecnológicos orientados a generar resultados amigables con el ambiente. El segundo, considerar a las organizaciones o conjunto de organizaciones como ecosistemas humanos orientados a la materialización de la innovación como cultura organizativa cuyo valor principal sea la sustentabilidad del ambiente.

Es decir, se le da una connotación ecológica de sustentabilidad, tanto al ambiente interno humano de la organización como al medio ambiente externo a través de la implementación de innovaciones de distinta naturaleza que lo favorezcan.

Obviamente, resaltar el tema "cooperación y redes para la innovación" fortalece la visión sistémica implícita en cualquier ecosistema, tanto humano como biológico, en el cual todos los componentes del sistema se apoyan unos con otros para lograr sus objetivos. Esto se convierte en una condición medular para la implementación de una cultura de innovación en las organizaciones y materializar la innovación con resultados tangibles e intangibles.

\section{Innovación. Una aproximación a su conceptualización}

Tal y como lo señalan Flores y Espósito (2014):

La innovación es un término elaborado a principios del siglo XX por el economista austríaco Joseph Schumpeter, tanto para dar una explicación a la sucesión 
de la crisis del capitalismo cada 50 años, como para abordar el problema de la dinámica interna profunda de este sistema económico. El uso inicial de la palabra innovación se reducía a la introducción de un objeto o métodos nuevos en el mercado. No obstante, la complejidad del término y de la realidad, ha permitido su extensión hacia otros tipos de fenómenos no relacionados directamente con los mercados, porque puede implicar tanto la idea de un cambio tecnológico generalizado, como un cambio social dentro de las organizaciones y la sociedad.

En este último aspecto señalado por Flores y Espósito, es donde haremos énfasis al presentar el cuadro 2 con distintas definiciones de innovación, las cuales destacan su dimensión intangible y complejidad, y confirman la presencia activa de procesos humanos clave que van de la mano para materializarla. Como se observa, se opta por conceptos de innovación amplios y abiertos.

\section{Cuadro 2}

Conceptualización de innovación

\begin{tabular}{|c|c|c|}
\hline Autor (es) & Concepto & $\begin{array}{l}\text { Procesos } \\
\text { humanos } \\
\text { asociados }\end{array}$ \\
\hline $\begin{array}{l}\text { BID-SECAB- } \\
\text { CINDA }(1994)\end{array}$ & $\begin{array}{l}\text { Avance del conocimiento humano aplicado efectivamente para } \\
\text { satisfacer necesidades de las personas o de organizaciones }\end{array}$ & $\begin{array}{l}\text { Aplicación del } \\
\text { conocimiento } \\
\text { humano }\end{array}$ \\
\hline Benavides (1999) & $\begin{array}{l}\text { Concepción sistemática y deliberada de nuevas ideas que, una vez } \\
\text { desarrolladas, permiten la introducción en el mercado de nuevos } \\
\text { productos o procesos, la adopción por parte de la empresa de } \\
\text { nuevas estructuras organizativas, el empleo de nuevas técnicas } \\
\text { comerciales, de gestión, entre otros. }\end{array}$ & $\begin{array}{l}\text { Concepción } \\
\text { sistemática y } \\
\text { deliberada de } \\
\text { nuevas ideas }\end{array}$ \\
\hline $\begin{array}{l}\text { Van de Ven et al } \\
\text { (1999) }\end{array}$ & $\begin{array}{l}\text { Proceso de desarrollo y aplicación de una nueva idea que puede } \\
\text { representar una recombinación de ideas viejas, un esquema que } \\
\text { cuestiona el orden actual, una fórmula o enfoque singular que sus } \\
\text { proponentes perciben como nuevo. }\end{array}$ & $\begin{array}{l}\text { Aplicación de } \\
\text { una nueva idea. } \\
\text { Recombinación de } \\
\text { ideas viejas. }\end{array}$ \\
\hline $\begin{array}{l}\text { Manual de } \\
\text { Frascati (2002) }\end{array}$ & $\begin{array}{l}\text { Conjunto de etapas científicas, tecnológicas, organizativas, } \\
\text { financieras, comerciales, incluyendo las inversiones en nuevos } \\
\text { conocimientos, que llevan o intentan llevar a la implementación de } \\
\text { productos y procesos nuevos o mejorados. }\end{array}$ & $\begin{array}{l}\text { Inversión en nuevos } \\
\text { conocimientos }\end{array}$ \\
\hline Miranda (2003) & $\begin{array}{l}\text { Resultado de la interacción comunicativa entre actores sociales } \\
\text { que realizan determinadas prácticas relevantes. }\end{array}$ & $\begin{array}{l}\text { Interacción } \\
\text { comunicativa }\end{array}$ \\
\hline $\begin{array}{l}\text { Sáenz y De } \\
\text { Souza (2006) }\end{array}$ & $\begin{array}{l}\text { Proceso de aprendizaje sistémico, interactivo, multidisciplinario y de } \\
\text { diferentes actores, en el cual nuevos conocimientos son generados, } \\
\text { otros son transferidos, otros ya existen en las organizaciones } \\
\text { participantes, otros son asimilados e interconectados para } \\
\text { introducir en la sociedad una nueva tecnología. }\end{array}$ & $\begin{array}{l}\text { Aprendizaje } \\
\text { sistémico, } \\
\text { interactivo, } \\
\text { multidisciplinario } \\
\text { y de diferentes } \\
\text { actores }\end{array}$ \\
\hline $\begin{array}{l}\text { Manual de Oslo } \\
(2006)\end{array}$ & $\begin{array}{l}\text { Introducción de un nuevo o significativamente mejorado, producto } \\
\text { (bien o servicio), de un proceso, método de comercialización } \\
\text { u organizativo, en las prácticas internas de la empresa, la } \\
\text { organización del lugar de trabajo o las relaciones exteriores. }\end{array}$ & $\begin{array}{l}\text { Prácticas internas } \\
\text { de la empresa, la } \\
\text { organización del } \\
\text { lugar de trabajo, } \\
\text { las relaciones } \\
\text { exteriores }\end{array}$ \\
\hline
\end{tabular}

Fuente: Elaboración propia 
La amplitud y complejidad de la innovación evidenciada en el cuadro anterior, ha conllevado a que se hayan establecido distintas tipologías, las cuales presentamos de forma resumida en la siguiente sección.

\section{Tipos de innovación}

El Manual de Oslo, además de explicar las innovaciones de producto y de procesos, incorpora las innovaciones de mercadotécnia y de organización (OCDE, 2006). Por su parte, Rodríguez y Alvarado (2008) incorporan la de tipo social y Ahmed et al, (2012) la innovación filosófica y la innovación política. Aun cuando los tipos de innovación, a excepción de la social, la filosófica y la política, están enfocados hacia las empresas, pueden perfectamente adaptarse a organizaciones de otra naturaleza, pero además veremos, cómo cada una de ellas son indiscutiblemente motorizadas por procesos humanos asociados con el aprendizaje, la interacción, la creación y aplicación de distintos tipos de conocimiento, lo que hace indispensable que tanto las organizaciones como grupos sociales que quieran innovar incorporen este enfoque en su lógica.

\section{a. Innovación de producto}

Se corresponde con la introducción de un bien o de un servicio nuevo, o significativamente mejorado, en cuanto a sus características $o$ al uso al que se destina. Esta definición incluye la mejora significativa de las características técnicas, de los componentes y los materiales, de la informática integrada, de la facilidad de uso u otras características funcionales. Las innovaciones de producto pueden, por un lado, utilizar nuevos conocimientos o tecnologías y por el otro, basarse en los ya existentes. El término producto cubre a la vez bienes y servicios. Las innovaciones de producto en los servicios pueden incluir mejoras significativas en la manera en que estos servicios se prestan (en términos de eficiencia o rapidez, por ejemplo), la adición de nuevas funciones o características a servicios existentes o la introducción de servicios enteramente nuevos. Cabe citar por ejemplo, la mejora de los servicios bancarios con el uso de la Internet.

\section{b. Innovación de proceso}

Es la introducción de un nuevo, o significativamente mejorado, proceso de producción o de distribución. Implica cambios significativos en las técnicas, los materiales y/o los programas informáticos y pueden tener por objeto disminuir los costos unitarios de producción o distribución, mejorar la calidad o producir o distribuir nuevos productos o sensiblemente mejorados.

Los métodos de producción pueden incluir técnicas, equipos y programas informáticos utilizados para producir bienes o servicios. Por ejemplo, instalación de un diseño asistido por computadora para la elaboración de un plano. Los métodos de distribución están vinculados a la logística de la organización y engloban los equipos, los programas informáticos y las técnicas para el abastecimiento de insumos, la asignación de suministros en el seno de la empresa o la distribución de productos finales.

Las innovaciones de proceso también puede incluir nuevos, o significativamente mejorados, métodos de creación y de prestación de servicios; 
la introducción de cambios significativos en los equipos y los programas informáticos utilizados por las empresas prestadoras de servicios 0 en los procedimientos o técnicas empleados para prestar dichos servicios como por ejemplo compras, contabilidad, cálculo o el mantenimiento. La introducción de una nueva o significativamente mejorada tecnología de la información y la comunicación es una innovación de proceso si está destinada a mejorar la eficiencia y/o la calidad de una actividad de apoyo básico. El producto final es el mismo, lo que cambia es cómo producirlo, lo que puede redundar en rapidez, optimización de materia prima, ahorro de recursos.

\section{c. Innovación de mercadotecnia}

Según el Manual de Oslo consiste en aplicar un nuevo método de comercialización que implica cambios significativos del diseño o envasado de un producto o su promoción. Las innovaciones de mercadotecnia tratan de abrir nuevos mercados o de posicionar en el mercado de una nueva manera un producto con el fin de aumentar las ventas. Lo que distingue la innovación de mercadotecnia de otros cambios en los instrumentos de comercialización de una empresa es la introducción de un método de comercialización que esta empresa no utilizaba antes. Esto debe representar una ruptura fundamental con relación a los métodos de comercialización ya practicados por la empresa. El nuevo método de comercialización puede haber sido puesto a punto por la empresa innovadora o adoptado de otra organización. La introducción de nuevos métodos de comercialización puede referirse tanto a productos nuevos como a los ya existentes.
La innovación de mercadotecnia también incluye cambios significativos en el diseño del producto son parte del nuevo concepto de comercialización y se remiten a cambios de forma y aspecto que no modifican las características funcionales o de utilización del producto. También incluyen modificaciones del envasado de los productos para los cuales el embalaje es el principal determinante de su aspecto; la introducción de cambios significativos en la forma, el aspecto o el sabor de productos alimentarios o bebidas. Los nuevos métodos de comercialización en materia de posicionamiento de productos se refieren a la creación de nuevos métodos de venta de bienes y servicios (no los de transporte, almacenamiento y manejo de productos) esencialmente relativos a la eficiencia. También implica la utilización de nuevos conceptos para la presentación y exhibición de productos.

\section{d. Innovación de organización}

Según el Manual de Oslo (2006) es la introducción de un nuevo método organizativo en las prácticas, la organización del lugar de trabajo - las relaciones de la organización con su entorno. Las innovaciones de organización en la práctica empresarial implican la introducción de nuevos métodos para organizar las rutinas y los procedimientos de gestión de los trabajos, para mejorar el aprendizaje y la distribución del conocimiento en la organización. Las innovaciones en la organización dellugarde trabajo implican la introducción de nuevos métodos de asignación de responsabilidades y del poder de decisión entre los empleados para la división del trabajo en el seno de los servicios y entre los servicios 
(y las unidades organizativas) de la empresa, así como nuevos conceptos de estructuración, en particular, la integración de distintas actividades. Las innovaciones organizativas pueden también implicar la centralización de las actividades y el refuerzo de la obligación de rendir cuentas con relación a las decisiones tomadas.

Los nuevos métodos de organización en materia de relaciones exteriores de una empresa implican la introducción de nuevas maneras de organizar las relaciones con las otras empresas 0 instituciones públicas, así como el establecimiento de nuevas formas de colaboración con organismos de investigación o clientes, de nuevos métodos de integración con los proveedores, y la externalización o la subcontratación, por primera vez, de actividades consustanciales a una empresa (producción, compras, distribución, contratación y servicios auxiliares).

Los cambios organizativos introducidos en respuesta a una nueva estrategia de gestión constituyen una innovación si representan la primera introducción de un nuevo método organizativo en las prácticas empresariales, la organización del lugar de trabajo o las relaciones exteriores. Hay innovación cuando se aplica la estrategia recurriendo a nuevos programas informáticos y métodos de recopilación de información para fomentar la puesta en común del conocimiento entre las distintas divisiones. Las fusiones $y$ adquisiciones en sí mismas no son innovaciones, pero pueden implicar innovaciones organizativas si la empresa elabora o adopta nuevos métodos de organización con motivo de estas operaciones.

\section{e. Innovaciones radicales y menores o incrementales}

Existe otra tipología de innovación asociada con su nivel de complejidad, que se puede dar tanto en procesos como en productos. Se trata de las innovaciones mayores o radicales y las innovaciones menores o incrementales. Las innovaciones mayores o radicales, se dan cuando hay cambios o saltos cualitativos que ameritan ser patentados. Las innovaciones menores, se dan cuando hay mejoras 0 incrementos reducidos. Para Shumpeter (1934), citado por Carrasco y Castaño (2008) las innovaciones radicales originan los grandes cambios del mundo, mientras que las innovaciones menores, incrementales o progresivas alimentan de manera continua el proceso de cambio.

\section{f. Innovación social}

Rodríguez y Alvarado (2008) señalan, y así también lo vemos, que las innovaciones sociales a menudo surgen donde ni el mercado ni los gobiernos han ofrecido alternativas a la población. Por lo tanto, es importante que los actores involucrados en el diseño y gestión de políticas públicas hagan esfuerzos adicionales por vincularse a estas innovaciones, facilitarlas y extraer de ellas lecciones útiles para diseñar o renovar la política pública.

$$
\text { Apoyándose en sus }
$$

investigaciones sobre innovaciones sociales, Rodríguez y Alvarado (2008) consideran que la mayoría de éstas son readaptaciones creativas en procesos y contextos distintos a los originales. En su mayoría tienen origen en soluciones alcanzadas por otro grupo 
en otro contexto, y su destino muy posiblemente sea seguir aplicándose en una escala superior, para beneficio de un mayor número de personas. $Y$ es en su réplica y multiplicación, que la innovación alcanza su pleno sentido porque le proporciona un sentido social.

Las principales fuentes

de la innovación social son las organizaciones de la comunidad, las no gubernamentales y las públicas. Generalmente las personas que participan en procesos de innovación social enfrentan dificultades para satisfacer sus necesidades básicas, ejercer sus derechos y protegerse creativamente frente a un sistema social estructuralmente excluyente.

Sin embargo, algunas veces no lo logra solo con sus propios medios, sino con un impulso adicional provisto generalmente por recursos externos: el grupo, la institucionalidad, la comunidad, el voluntariado, la intercooperación, las alianzas -informales o contractualesprincipalmente con el sector público, y los intercambios no mercantiles de información. Las innovaciones que se originan y difunden en el circuito mercantil tienen por incentivo la ganancia, intrínseca al sistema de precios. En el caso de las innovaciones sociales el incentivo tiene un valor intangible: el de la esperanza o dignidad recuperada, la certidumbre de que un mundo mejor, fundado en la solidaridad y la cooperación, siempre es posible.

\section{g. Innovación filosófica}

Ahmed et al, (2012) señalan este tipo de innovación aparentemente está alejada del mundo empresarial, sin embargo, el nuevo pensamiento filosófico al tener un efecto significativo en la sociedad y en la forma en que ésta se administra y conduce a sí misma, las empresas se verán involucradas en sus efectos. El pensamiento filosófico orienta a la sociedad al suministrar conocimiento de vanguardia y a definir lo que es correcto y lo que es incorrecto. Los autores ponen como ejemplo la revolución filosófica en Rusia porque la transición del socialismo hacia la aceptación del capitalismo de mercado abrió un mercado de más de 1700 millones de consumidores. Por lo general comienza con el dinamismo de la filosofía antes de su aceptación por parte de instituciones sociales, agentes u organizaciones de influencia.

\section{h. Innovación política}

Ahmed et al, (2012) la definen como los cambios en el escenario político que a menudo tienen consecuencias importantes en términos de la dirección y el desarrollo de la sociedad, así como de las organizaciones. Las innovaciones políticas con mayor frecuencia toman la forma de legislación, reformas institucionales, dirección social y gobierno. Indican los autores que Japón es un ejemplo de la influencia de la innovación política sobre la arena corporativa debido a que después de la Segunda Guerra Mundial, el gobierno japonés promulgó un entorno legal novedoso, caracterizado por la confidencialidad de los acuerdos de apoyo institucional del gobierno. Las corporaciones japonesas emergieron gracias a la política industrial del Ministerio Japonés de Industria y Comercio y del Ministerio de Finanzas bajo la figura de acuerdos que dieron apoyo a la transferencia de tecnología 
occidental y de métodos gerenciales para las corporaciones japonesas. Esto fue la clave para sentar los cimientos del crecimiento económico y prosperidad del Japón.

\section{La cultura de innovación}

Rodríguez (1997) plantea que a pesar de la complejidad de las exigencias del entorno de las organizaciones, predominan las respuestas de tipo cuantitativo (Ejemplo: reducción de costos de factores de producción) sobre las de tipo cualitativo (Ejemplo: aprovechamiento de la capacidad innovadora del talento humano). González (2013) señala que es importante medir los resultados de la innovación para conocer sus avances, aportes a la organización (crecimiento, costos, eficiencia, entre otros) y la ruta hacia los objetivos que se persiguen. No obstante, Rodríguez considera que enfocarse solo en lo cuantitativo se opone a la expansión de lo cualitativo en las organizaciones si se convierte en el enfoque predominante. Pero, no se trata de contraponer lo cuantitativo a lo cualitativo, porque ambos se complementan, sino de evitar el desequilibrio entre las posibilidades reales de los dos.

Cuando hablamos de cultura en las organizaciones nos referimos a aquellos comportamientos que surgen de manera natural, espontánea, sin que nadie nos lo imponga ni nos recuerde que lo tenemos que hacer porque forma parte intrínseca de nuestro ser, de nuestra forma de trabajar y de vivir. En este artículo asumiremos la definición de Cornejo y Muñoz (2009) de cultura de innovación como el conjunto de conocimientos, prácticas y valores (individuales y colectivos), que determinan disposiciones y formas de hacer las cosas y que promueven, en la sociedad, la generación de nuevos conocimientos y la creación de innovaciones.

Cada organización tiene su propia cultura constituida por determinados valores y actitudes que determinan comportamientos, facilitarán u obstaculizarán los procesos innovadores. Es allí donde los gerentes y directivos deben estar alerta.

Por tal razón, es que los cambios de cultura organizacional pueden considerarse difíciles e incluso imposibles, por la tendencia natural de hacer las cosas como siempre la hemos hecho si nos sentirnos cómodos y hemos tenido éxito con ellas. Pero cuando el entorno cambia, es prácticamente inevitable que las personas tengan que cambiar sus formas de trabajar si quieren que la organización donde laboran permanezca en el tiempo y se posicione como un sólido referente de éxito. Para lograrlo, no es suficiente poseer una infraestructura tecnológica y física de avanzada ni tampoco ingentes recursos económicos. Es determinante una cultura de innovación, con un fuerte respaldo de su directiva.

Aun cuando los países y empresas más innovadores son los que mayores medios financieros y productivos tienen, también existen casos que matizan la importancia del factor económico como único motor de la innovación. Así, por ejemplo, Nonaka y Takeuchi (1995) señalan que el desarrollo de la industria japonesa después de la II Guerra Mundial, se debió principalmente, a una nueva forma de entender el valor estratégico del conocimiento para su gobierno pero también para las empresas. 
Por su parte, Cornejo y Muñoz Ruiz (2009) señalan que en España, desde un punto de vista empresarial, una pequeña compañía textil de batas llamada Goa de La Coruña, dentro de un entorno poco desarrollado, ha podido convertirse en el imperio textil mundial llamado Inditex. Los ejemplos anteriores además de los planteamientos que hemos hecho en secciones previas de este artículo, sustentan el papel protagónico del factor humano como elemento medular que fundamentado en actitudes innovadoras y creativas son los que van a potenciar los recursos tangibles, generalmente escasos, para materializar proyectos de innovación.

\subsection{Obstáculos para el establecimiento de una cultura de innovación en las organizaciones}

A pesar de las bondades de la cultura de innovación para las organizaciones y países, persisten obstáculos que dificultan su adecuado desarrollo. Nos interesan a efectos de este artículo, los asociados directamente a los procesos humanos. El Manual de Oslo, por ejemplo, indica: falta de personal cualificado con conocimientos para desarrollar innovaciones, personas que pueden desarrollar conductas resistentes al cambio o a los productos 0 procesos innovadores, la presión del día a día, castigar los errores.

Por su parte, Quintanilla (2000) precisa la existencia de factores culturales, como por ejemplo, creencias religiosas; y sociedades con bajo nivel de formación científica y técnica, mientras que para Keley (2006), existen organizaciones desinteresadas en cambiar la forma de hacer las cosas, se valora el pensamiento crítico más que el pensamiento creativo, se castigan los errores y hay negación de aprender de ellos, innumerables permisos y aprobaciones para probar nuevas ideas, robo de ideas para progresar, para ascender en la organización siempre hay que seguir los manuales, los que osan probar formas distintas de hacer las cosas y se equivocan, son escarmentados ejemplarmente.

A pesar de estos obstáculos, también hay formas de estimular la cultura de innovación en la organización. Las cuales las veremos en la siguiente sección.

\subsection{Estímulos para establecer una cultura de innovación en la organización}

De acuerdo con Keley (2006), las propuestas para fomentar la cultura de innovación, se orientan a: premiar colectivamente los logros innovadores, estimular la búsqueda de nuevas formas de hacer las cosas, así como los aportes creativos para evitar que la inercia del grupo los detenga.

El autor también propone reforzar valores y actitudes de apertura al cambio; promover que los problemas sean vistos como retos; difundir internamente los aportes que hayan tenido un impacto positivo en el desempeño de la organización y reconocerlos públicamente; estimular la difusión del conocimiento, incluido aquel que se obtiene en los fracasos; permitir las fallas cuando éstas son consecuencia inevitable del proceso de experimentación, valorar la capacidad de desarrollar a los integrantes del equipo; retar a los empleados a encontrar mejoras en todos los procesos; castigar 
la reacción crítica ante nuevas ideas y a la vez de estimular la integración de diferentes formas de pensamiento.

Para González (2013) las claves que permitirán a una organización, independientemente de su tamaño, evolucionar hacia una cultura de innovación valoran las personas con mayor capacidad de influencia en la organización que creen en la innovación y la lideraran; disponen de un sistema de gestión para la innovación, mantienen una perspectiva transversal de la innovación, que se focalice tanto en la fase de creatividad, como de implantación; hacen de la innovación una forma habitual de trabajo en la organización, no un proceso aislado y cuentan con todas las personas de la organización, aportando, desarrollando y experimentando ideas.

Asimismo, para el autor también se requiere para fomentar o fortalecer la cultura de innovación en las organizaciones que en éstas se visualice la creatividad como uno de los ejes clave para la innovación y que ésta pueda surgir de cualquiera de los miembros de la organización; se identifiquen personas dispuestas a cambiar su forma de trabajar; se arriesguen a buscar nuevas formas de hacer las cosas y trabajar con ellas para desarrollar sus capacidades de innovación; se integren equipos de personas con experiencias y visiones distintas que puedan producir debates para enriquecer el proceso de innovación; se delegue y dote de capacidad de decisión a los empleados con el fin de crear una relación de confianza entre directivos y empleados; se mantengan altos niveles de tolerancia para asumir riesgos; no se penalice el fracaso sino que se gestione como parte del proceso de experimentación; se aprovechen las TIC's para generar entornos colaborativos, dentro y fuera de la organización, y se implementen reconocimientos al personal a través de premios, retribuciones, desarrollo profesional o promociones.

Los planteamientos anteriores evidencian, entre otros aspectos, que para incorporar o sostener la cultura de innovación en cada una de las personas que laboran en la organización se requiere de un estilo de liderazgo que fomente la participación en la toma de decisiones; clima de confianza; alineación de los procesos de gestión de recursos humanos con el desarrollo del talento humano; motivación y compromiso de los empleados hacia la innovación; sistema de incentivos (no necesariamente monetarios) de estímulo a la innovación; comunicación abierta con los empleados; consenso de ideas en la búsqueda de nuevos caminos para aceptar y asumir la necesidad de cambios y así implementarlos eficientemente; visión del error como oportunidad de aprendizaje y no como fracaso.

\section{Conclusiones}

La innovación supone un entramado de interrelaciones que requieren de actores diversos en permanente reorganización. La innovación es un fenómeno complejo en proceso de estudio y evolución en función de un entorno igualmente complejo que ha abierto la posibilidad de analizarla desde distintos enfoques. Por lo tanto, es multi causal así como lo es la cultura de innovación, comportamiento humano que tiene repercusión en las organizaciones y en el país donde se lleve a cabo. Por tal motivo, necesita sustentarse en condiciones favorables de distinta naturaleza como jurídicas, 
organizativas, financieras y políticas, pero sobre todo humanas.

Facilitar las condiciones para que se genere una cultura de innovación en la gente en todos los niveles de la organización es responsabilidad de su directiva pero en plena comunicación con todo el personal de manera sinérgica.

La innovación puede darse en cualquier organización, independientemente de la actividad que realice, no necesariamente en organizaciones con tecnología de punta; puede no estar orientada hacia el mercado como la innovación social ni ser únicamente responsabilidad del departamento de I+D+I. La innovación debe convertirse en la cultura de la organización, en un proceso estratégico asociado a lo social y lo cultural de toda la organización, y al compromiso con la preservación del medio ambiente en el cual está inmersa con el fin de fomentar en su gente la responsabilidad participar activamente en la construcción de un mundo mejor basado en la cooperación con otros. Es allí donde entran a jugar un importante papel, no solo los conocimientos formales y económicos, sino también lo social, lo organizativo, lo cultural y las redes de relaciones entre los agentes de innovación.

Esto implica una tarea ardua que tiene que ver con cambios de comportamiento, formas de pensar, acciones a seguir, hábitos, rutinas, patrones de trabajo y prioridades. Pero como la innovación es un proceso humano que no es fácil de asumir, es importante que los responsables de llevarlo a cabo, sean capaces de hacerle entender a su equipo de trabajo las razones que lo motivan, permitirles procesar dicho cambio, aportar sus ideas y expresar sus temores, en fin, acompañarlos de manera sincera en el proceso. Esta será la diferencia entre que prospere o fracase la incorporación de cambios en las organizaciones.

La estructura de la organización puede influir en la calidad de los resultados del trabajo innovador. Mientras más flexible sea la estructura organizativa y menos rígidos sean los procesos mejorará la coordinación de acciones que favorezcan la implementación de estrategias de innovación. Esta flexibilidad se asocia con la posibilidad de que empleados de distintos departamentos puedan migrar libremente a otros para intercambiar ideas y generar proyectos de manera conjunta; mejorar en la integración organizativa y aumentar los niveles de autonomía del personal en la toma de decisiones.

Esto favorece el interés por el aprendizaje, el rediseño de rutinas de trabajo y prácticas organizacionales lo suficientemente flexibles como para adaptarse a la complejidad del entorno, a la organización en sí misma y a las relaciones dentro y fuera de ella. Transformarse en una organización fluida y flexible motiva a los individuos a formular nuevas ideas y procedimientos que a futuro se pudieran materializar en productos y servicios exitosos. Todo esto requiere tanto del personal como de sus directivos una actitud abierta hacia la innovación y una mentalidad flexible como la organización a la cual se quiere llegar.

Esto nos llevaa loque anteriormente comentamos sobre ecosistemas de innovación y cooperación y redes para la innovación. Para que se implemente exitosamente la cultura de innovación en la organización es importante pensar en red, no como entes aislados. Es transcendental tener conciencia de que formamos parte de otros sistemas 
que se ven influenciados por nuestras acciones y nosotros por las de ellos. Lo más interesante de este cambio de visón es que percibamos a esos sistemas externos al nuestro como aliados con los cuales podemos cooperar para innovar. Es ese ecosistema humano al que debemos vincularnos y escuchar para fomentar valores que permitan incorporar innovaciones en la organización lo más armónica y sustentablemente posible con el entorno.

\section{Referencias bibliográficas}

Ahmed, Pervaiz; Shepherd, Charles; Ramos, Leticia y Ramos, Claudia (2012), Administración de la innovación, México: Pearson.

Asociación Latinoiberoamericana de Gestión Tecnológica (2011), Memorias del XIV Seminario ALTEC, Lima. Perú.

Asociación Latinoiberoamericana de Gestión Tecnológica (2009), Memorias del XIII Seminario de ALTEC Ediciones Tecnológicas de Bolívar: Cartagena de Indias. Colombia.

Asociación Latinoiberoamericana de Gestión Tecnológica (2007), Memorias del XII Seminario ALTEC, Buenos Aires. Argentina.

Asociación Latinoiberoamericana de Gestión Tecnológica (2005), Memorias del XI Seminario ALTEC, Salvador. Brasil.

Asociación Latinoiberoamericana de Gestión Tecnológica (2003), Memorias del X Seminario. ALTEC, México, D.F.

Asociación Latinoiberoamericana de Gestión Tecnológica (2001), Memorias del IX Seminario. ALTEC, San José. Costa Rica.

Asociación Latinoiberoamericana de Gestión Tecnológica (1999), Memorias del VIII. ALTEC, Valencia. España.

Asociación Latinoiberoamericana de Gestión Tecnológica (1997), Memorias del VIII Seminario ALTEC, La Habana. Cuba.
Asociación Latinoiberoamericana de Gestión Tecnológica (1995), Memorias del VI Seminario ALTEC, Concepción. Chile.

Asociación Latinoiberoamericana de Gestión Tecnológica (1993), Memorias del V Seminario ALTEC, Bogotá. Colombia.

Asociación Latinoiberoamericana de Gestión Tecnológica (1991), Memorias del IV Seminario ALTEC. Caracas. Venezuela.

Benavides, Carlos (1998), Tecnología, innovación y empresa. Madrid, España: Editorial Pirámide.

BID-SECAB-CINDA (1990), Glosario de términos de gestión tecnológica. Colección Ciencia y Tecnología $N^{\circ}$. 28. Santiago de Chile

Carrasco, Inmaculada y Castaño, María Soledad (2008), El emprendedor Schumpeteriano y el contexto social. Revistas de Información Comercial Española (ICE) (845), 121134. Noviembre-diciembre. Pág. 1. Disponible en http://www.revistasice. com/cachepdf/ICE 845 121-134 F6565B36FDFD765A932BB8BEE $\overline{E 9}$ AAB41.pdf

Cornejo Cañamares, María y Muñoz Ruiz, Emilio (2009), Percepción de la innovación: cultura de la innovación y capacidad innovadora. Pensamiento Iberoamericano $\mathrm{n}^{\circ} 5$. pp 121-147. Disponible en: igital. csic.es/bitstream/10261/35048/1/ Percepcion_innovacion.pdf

Flores Urbáez, Matilde y Espósito de Díaz, Concetta (2014), Fundamentos básicos de gestión de la tecnología: teoría y práctica. Ediciones Vicerrectorado Académico. Universidad del Zulia. Maracaibo. Venezuela.

González, Coral (2013), ¿Cómo implantar la cultura de la innovación en la empresa? Entrevista. Disponible en http://www.peoplematters. com / A r chivos / D e s cargas / Apariciones\%20PM\%20Papel/2013/ Febrero/1130208_La\%20Razon.pdf 
González; Domingo; Sbragia, Roberto; Galante; Oscar; Soto, Rocío y Valdivieso, Renato (2013), ALTEC y la Gestión Tecnológica en Iberoamérica: Investigación y Gestión en las Empresas. Journal. Technology. Management. Innovation. Volumen. 8. ISSN: 0718-2724. Universidad Alberto Hurtado, Facultad de Economía y Negocios. Chile. Disponible en: (http://www.jotmi.org)

Kelley, Tom (2006), IDEO, La reina de la innovación. Entrevista. Revista Dinero, especial Innovación, Octubre 27. Disponible en http:// www.innovatividad.net/documentos/ Cultura_de_Innovacion.pdf

Miranda, Byron (2003), Capital social, institucionalidad y territorios: EI caso de Centroamérica, Nicaragua y Costa Rica. Instituto Interamericano de Cooperación para la Agricultura (IICA). Servicio de Información Mesoamericano sobre Agricultura Sostenible (SIMAS). Nicaragua y Costa Rica. Disponible en http://orton. catie.ac.cr/repdoc/A5187E/A5187E. PDF

Nonaka, Ikujiro y Takeuchi, Hirotaka (1995), The knowledge Creating Company. Nueva York, Oxford University Press.

Organización de Cooperación y Desarrollo Económicos -OCDE- (2002), Manual de Frascati. Propuesta de norma práctica para encuestas de investigación y desarrollo experimental. España. Disponible en http://www.idi.mineco. gob.es/stfls/ MICINN/Investigacion/FICHEROS/ ManuaFrascati-2002_sp.pdf

Organización de Cooperación y Desarrollo Económicos - OCDE- (2006), Manual de Oslo. Guía para la recogida e interpretación de datos sobre la innovación. Oficina de Estadística de la Comunidad Europea (EUROSTAT). Disponible en www.tragsa.es y en http://www.micit.go.cr/index.php/ docman/doc_details/95manualdeoslo. Html

Quintanilla, Miguel Ángel (2000), “Técnica y Cultura." Revista Teorema volumen XVII/3 1998, Filosofía y Tecnología. Edición electrónica. Agosto 2000.

Rey, Nohora y Tandredy, Francisco (2008), De la innovación social a la política pública. Historia de éxito en América Latina y el Caribe. Comisión Económica para América Latina y el Caribe (CEPAL). Naciones Unidas. Disponible en http://www. cepal.org/publicaciones/xml/2/41582/ innovacion-social-politicapublica-2010.pdf

Rodríguez Cortezo, Jesús (1997), Tecnología e industria: realidades alcanzables. Colección Libros para el Debate. Escuela de Gestión Comercial y Marketing. Madrid.

Rodríguez Herrera, Adolfo y Alvarado Ugarte, Hernán (2008), Claves de la innovación social en América Latina y el Caribe. Comisión Económica para América Latina y el Caribe (CEPAL), noviembre, Santiago de Chile. Disponible en http://www. cepal.org/publicaciones/xml/2/34682/ Claves_de_innovacion_social.pdf.

Sáenz, Tirso y De Souza, María Carlota (2006), Innovación tecnológica y sustentabilidad. En: Gestión de la Innovación: una visión actualizada para el contexto iberoamericano. Editores: Faloh, Rodolfo y Fernández, María Candelaria. Red Iberoamericana de Centros de Apoyo a la Innovación, La Habana, Cuba: Editorial Academia.

Van de Ven, Andrew; Polley, Douglas; Gaud, Raghu y Shankaram, Venkataraman (1999), EI viaje a la innovación. EI desarrollo de una cultura organizacional para innovar, México: Editorial Oxford University Press. 